\title{
Control for Microgrids with Inverter Connected Renewable Energy Resources
}

\author{
Hemanshu R. Pota \\ SEIT, UNSW@Canberra \\ Canberra ACT 2600 \\ Australia \\ h.pota@adfa.edu.au
}

\author{
M. J. Hossain \\ Griffith University \\ Gold Coast, QLD 4222 \\ Australia \\ j.hossain@griffith.edu.au
}

\author{
M. A. Mahmud \\ Swinburne University \\ Hawthorn, VIC 3122 \\ Australia \\ mmahmud@swin.edu.au
}

\author{
Rajit Gadh \\ University of California \\ Los Angeles, CA 90095 \\ USA \\ gadh@ucla.edu
}

\begin{abstract}
This paper contains a control scheme for power sharing in islanded microgrids with inverter-sourced distributed energy resources that combines robust control and droop control. As the load within the microgrid changes, the inverter-sourced generators will share this change in load. This paper includes a background on control schemes for power sharing and highlights the difficulty in exact reactive power sharing. In this paper a Distributed Energy Resource (DER) unit consists of an energy source and an energy storage system. A robust control scheme is proposed for the energy source which is augmented by droop control for the storage system. The performance of the proposed controller is demonstrated using a test microgrid system.
\end{abstract}

Index Terms-Microgrid, power balance, energy storage, renewable energy source

\section{INTRODUCTION}

Interconnection of microgrids is a practical way to achieve higher utilisation of renewable energy, reduce transmission losses, lower infrastructure capital investment, and achieve higher reliability of electricity supply [1], [2]. Experimental microgrids have been developed to test the basic ideas [3].

Energy sources in microgrids are likely to be renewable resources interconnected via voltage source converters (VSC). Most VSCs are controlled to output a set voltage magnitude and phase. This is in contrast to synchronous machine based grid operation where the voltage magnitude and the rate-ofchange of the generator angle (frequency) is set as a result of the interaction between the generation and load dynamics. As shown in the following if the VSC output voltage magnitude and angle are not controlled the system operation will result in unplanned generation levels and voltage profile in microgrids [2].

There exists rich literature in power sharing amongst parallel inverters [4]. It is common to refer to real power sharing droop control as frequency droop control [4]. The frequency droop control is so described that the output of each inverter has a different frequency. In power systems operation it is assumed that there exists a steady-state frequency and thus each inverter output cannot be at different frequencies. In this paper the frequency droop control is stated in terms of the output angle of the inverters. Present day fast acting inverters are able to quickly change the phase of the output voltage and it is more meaningful to specify the power sharing control as a rate-ofchange of angle droop control.
Recent survey papers [5], [6] contain useful background information on microgrids and a rich bibliography. A review of experimental microgrids is covered in [7]. The sharing of the reactive power in a microgrid is complicated owing to the fact that the conventional method of sharing based on the generator terminal voltage does not work in microgrids [8]. Some solutions for reactive power sharing from the literature are discussed in this paper.

Most of the microgrid literature treats voltage sources behind the inverters as ideal [1], [2], [4]. This assumption is perfectly valid for the research in power sharing amongst parallel connected inverters [4] but it needs extension when the ideal voltage sources are replaced with renewable energy resources. The presentation in this paper is with an emphasis on microgrids from a power systems point-of-view. It is clearly brought out that the rate-of-change of angle droop requires a change in the input power and there needs to be another controller to achieve this objective. In the next section an analysis is presented which brings out the essential elements in control algorithms for power sharing amongst renewable resources in microgrid.

\section{Power Sharing With Control}

The control methods used for power sharing can be broadly classified into two categories. The first category is of masterslave control methods that have been developed for load sharing by parallel connection of uninterrupted power supplies. The second category is of droop control methods which are more suited to load sharing among DERs in microgrids. A summary of both the categories is presented next.

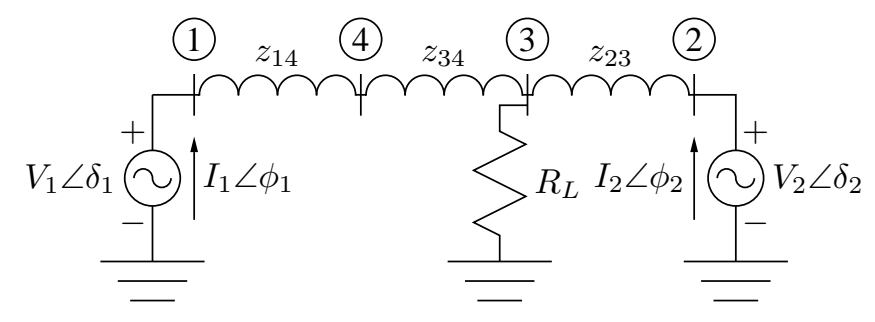

Fig. 1. A Simple Microgrid 


\section{A. Droop Control}

In this section the droop control method for sharing power due to load changes is presented. For inverter-sourced generation the phase of the voltage can be advanced according to a control law which appears like a droop. For example, angle $\delta_{i}$ is reduced if the generator supplies more than the reference load and vice-verse. The droop controllers for the two energy sources shown in Figure 1 are $(i=1,2)$ :

$$
\dot{\delta}_{i}=-k_{p_{i}}\left(P_{m_{i}}-P_{i}^{0}\right)
$$

and

$$
\Delta V_{i}=-k_{q_{i}}\left(Q_{m_{i}}-Q_{i}^{0}\right) .
$$

where for source $i, P_{i}^{0}, Q_{i}^{0}$ are the set-point and $P_{m_{i}}, Q_{m_{i}}$ are the measured real and reactive output power; $k_{p_{i}}, k_{q_{i}}$ are droop constants; $\Delta$ is to represent small changes.

For a stable operation it is necessary that the real and reactive powers are such that $\dot{\delta}_{1}-\dot{\delta}_{2}=0$. This guarantees that using the droop control law (1), the real power is shared in inverse proportion to $k_{p_{i}}$. In steady-state the change in the system frequency is $\dot{\delta}_{1}=\dot{\delta}_{2}=\Delta \omega$. The QV droop control in (2) does share the reactive power change but it does not share it proportionately and it depends on transmission line parameters. Next we present a two-bus system analysis with a view to designing a droop scheme to share the change in the reactive power proportionately.

\section{B. Reactive Power Sharing using Communication}

For a two-source microgrid shown in Figure 1, for a change in $\Delta Q_{3}$, the ratio of the change in $\Delta Q_{1}$ and $\Delta Q_{2}$ is given as follows:

$\frac{\Delta Q_{1}}{\Delta Q_{2}}=\frac{-V_{1}^{0} B_{13} \cos \delta_{13}^{0}\left(1-k_{q_{2}}\left(V_{3}^{0} B_{23} \cos \delta_{23}^{0}+2 V_{2}^{0} B_{22}\right)\right)}{-V_{2}^{0} B_{23} \cos \delta_{23}^{0}\left(1-k_{q_{1}}\left(V_{3}^{0} B_{13} \cos \delta_{13}^{0}+2 V_{1}^{0} B_{11}\right)\right)}$

Expression (3) clearly indicates that the sharing of reactive power using a simple droop will be in the ratio of admittances. This is a fundamental limitation in the use of $\mathrm{QV}$ droop control. In the following we discuss how to overcome this limitation and provide proportional reactive power sharing.

In [9] virtual impedance concept for reactive power sharing and resonant filters for harmonic current support is proposed. The control scheme [9, Fig. 11] shows how the measured current is used with an "impedance" block to synthesise virtual impedance.

In [10], [11] the design of decentralised robust controllers for multi-DER microgrids is presented. Each DER is a subsystem with a fully controllable voltage source and the control objective being the magnitude and angle of the voltage at the Point of Common Coupling (PCC). The PCC voltage settings are communicated from a central power management system using power flow analysis.

In [12] the communication amongst voltage controllers is achieved using higher frequency voltages $\left(v_{c_{i}}, i=\right.$ $1,2, \ldots, N)$ superimposed on the power frequency voltage sources as shown in Fig. 2. The rate of change of the phase of $v_{c_{i}}$ is set proportional to $\Delta Q_{i}$ and $\Delta V_{i}=-k_{c_{i}} \Delta P_{c_{i}}$. The change in the transfer of the real power at the communication

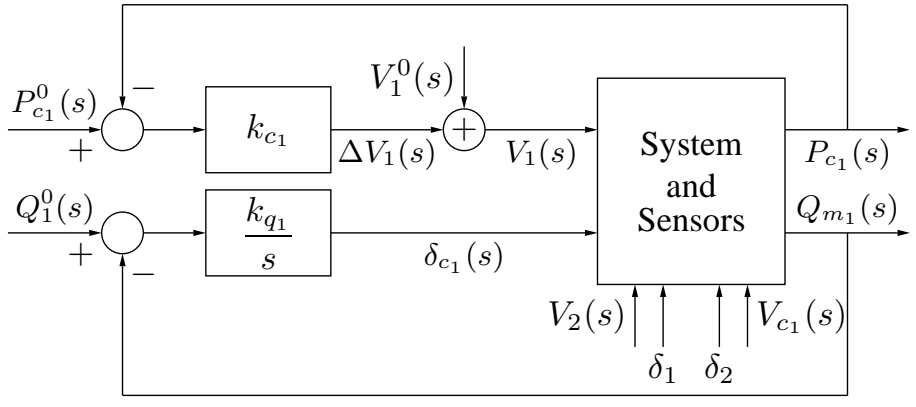

Fig. 2. Droop control for reactive power sharing with communication

frequency is measured and the rate of change of the phase of $v_{c_{i}}$ continues until all the rates are equal and there is no change in the real power transfer at the communication frequency. Once this is achieved equal $\Delta V_{i}$ is guaranteed thus proportionate reactive power sharing.

In [13], a two stage droop control is suggested. The first stage is the conventional droop control,

$$
\begin{aligned}
& \omega=\omega_{0}-D_{P} P \\
& E=E_{0}-D_{Q} Q
\end{aligned}
$$

A second stage controller action is initiated every few seconds synchronously by all the DER controllers on receiving a communication from the central controller. The controller parameters are tuned to complete the control operation in a few milli-seconds,

$$
\begin{aligned}
& \omega=\omega_{0}-\left(D_{P} P+D_{Q} Q\right) \\
& E=E_{0}-D_{Q} Q+\left(\frac{K_{C}}{s}\right)\left(P-P_{\mathrm{AVE}}\right)
\end{aligned}
$$

where the measured power $P_{\mathrm{AVE}}$ is held constant during the second stage.

In [14] a robust droop controller is presented for a predominantly resistive network where reactive power is controlled by varying the phase difference and the real power by voltage magnitude. A proportionate sharing is achieved much like (11) introduced in [8].

The difficulty with exact reactive power sharing as compared to real power sharing amongst multi-DER microgrids is that it is difficult to use an integral control with reactive power sharing. Let us look at the droop control equations (1)-(2), the system reaches equilibrium only when all $\dot{\delta}_{i}$ are equal but the same system has stable operation for multiple combinations of $\Delta V_{i}$. A proportionate reactive power sharing can be achieved only when the control algorithm can guarantee equal values of all $\Delta V_{i}$ at the new equilibrium.

It is possible but a very difficult problem to design control algorithms to ensure

$$
\Delta V_{1}=\Delta V_{2}=\cdots=\Delta V_{N}
$$

One way to achieve equal $\Delta V_{i}$ is to have an integral control of the form:

$$
\Delta V_{i}=\int\left(\Delta V_{i}-\Delta V_{\mathrm{com}}\right) d t
$$


where $\Delta V_{i}=-k_{q_{i}}\left(Q_{m_{i}}-Q_{i}^{0}\right)$ and $\Delta V_{\text {com }}$ has to be the change in the voltage at a pre-chosen common point in the microgrid and this scheme needs communication amongst the inverters or the DERs.

In [15] the control law is:

$$
\begin{aligned}
V_{i} & =V_{i}^{0}+k_{q i_{i}} \int\left(V_{\text {ref }_{i}}-V_{\text {com }}\right) d t \\
V_{\text {ref }_{i}} & =V_{i}^{0}-D_{q_{i}} Q_{i} .
\end{aligned}
$$

In [8] a proportional term is added to the above control law (9)(10):

$$
\begin{aligned}
V_{i} & =V_{i}^{0}+k_{q p_{i}}\left(V_{\mathrm{ref}_{i}}-V_{\mathrm{com}}\right)+k_{q i_{i}} \int\left(V_{\mathrm{ref}_{i}}-V_{\mathrm{com}}\right) d t \\
V_{\mathrm{ref}_{i}} & =V_{i}^{0}-D_{q_{i}} Q_{i} .
\end{aligned}
$$

In the two-source microgrid considered in this paper $V_{\text {com }}=$ $V_{3}$.

When the system is stable, the argument of the integral in (11) should be zero, thus:

$$
V_{i}^{0}-D_{q_{i}} Q_{i}=V_{\text {com }} .
$$

If voltages $V_{i}^{0}$ are the same then the reactive power is shared inversely proportional to $D_{q_{i}}$. Moreover the voltage change for $V_{\text {com }}$ will depend on the values of $D_{q_{i}}$. For small changes in $V_{\text {com }}$, constants $D_{q_{i}}$ must be small. The constants $k_{q i_{i}}$ determine the speed of response.

In this paper communication is used to transmit the results of a centralised optimal load-flow to individual DERs to achieve a desirable power sharing. The set values are achieved using a robust controller and the transient power balance is achieved using the local storage available with each DER unit.

\section{Control Strategy for Microgrids With DERS}

There are different ways to choose the reference real and reactive power $P_{i}^{0}, Q_{i}^{0}$ values for DERs. In this paper the values prior to load change are used as the reference. In [16] the DC-link voltage in a Photovoltaic (PV) system is used to set the reference real power. In [17] a virtual impedance scheme is introduced to achieve a balance in (a) reactive power sharing, and (b) harmonic current sharing, amongst many voltage-sources. Nonlinear loads have to be supplied with harmonic currents thus there is a need to share the required harmonic currents equally amongst various voltagesources. In [4] a modified active and reactive power $P^{\prime}$ and $Q^{\prime}$ are proposed for control. This paper [4] also discusses the use of virtual impedance for droop control. Future research needs to consider the dynamics of the energy resources along with the sensor dynamics for a proper design of microgrid droop controllers.

In [18] a wind generator is combined with a storage device to provide a smooth output power. The wind generator is modelled with its dynamic equations but the energy storage devices are modelled as ideal DC sources. Reference power output for the wind generator is obtained by using short-term predictions of wind speeds and pitch angle control. Fuzzy control is employed to control the pitch angle.

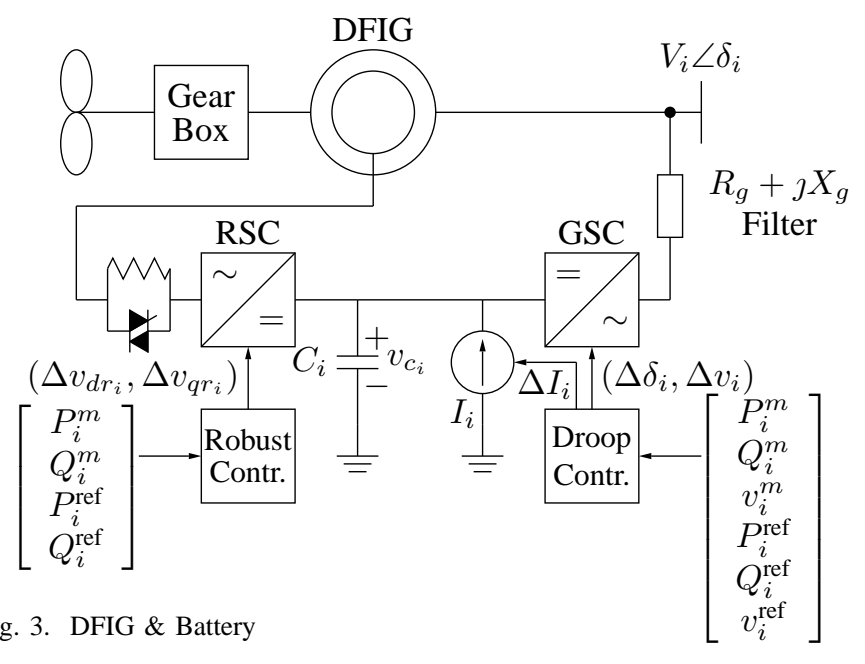

Robust control methods for controlling solar PV [19] and wind generation [20] have been proposed for transmission and distribution systems [21]. In this paper these robust control algorithms are extended to work in the microgrid framework by appending an energy storage system and a droop controller to each DER.

In this paper the problem of reactive power sharing is divided into long-term sharing and short-term sharing. For the long-term sharing a centralised system communicates reference values to each DER and short-term sharing is done using droop control of energy storage systems. Next we describe two DER units, one with a wind generation and another with solar PV system.

\section{A. DFIG and BES Control}

A Doubly-Fed Induction Generator (DFIG) with a battery energy storage (BES) (represented as a current source) is shown in Fig. 3. Droop control and energy storage system control for the DFIG is achieved using the following control laws (superscript $m$ is used for measured values and $w$ for wind turbine, positive $\Delta I_{w}$ indicates battery charging):

$$
\begin{gathered}
\Delta I_{w}=-k_{I_{w}} \int\left(v_{d c}^{\mathrm{ref}}-v_{d c}^{\mathrm{m}}\right) d t \quad \text { (Battery Control) } \\
\Delta \delta_{w}=k_{\delta_{w}} \int\left(P_{w}^{\mathrm{ref}}-P_{w}^{\mathrm{m}}\right) d t \\
\Delta v_{w}=k_{q_{w}} \int\left(Q_{w}^{\mathrm{ref}}-Q_{w}^{\mathrm{m}}\right) d t
\end{gathered}
$$

In general $P_{w}^{\mathrm{ref}}$ will be the predicted maximum power point based on wind speed and $P_{w}^{\text {ref }}$ and $Q_{w}^{\text {ref }}$ are achieved using the robust controllers in [20]. The desired active and reactive power is realized by controlling $i_{q r}$ and $i_{d r}$. A checker block checks the available capacity of $i_{q r}$ with respect to $i_{d r}$ so as not to exceed the limits of operation. The battery, shown as a current source in Figure 3, charges or discharges to keep the capacitor voltage set to a reference value. 


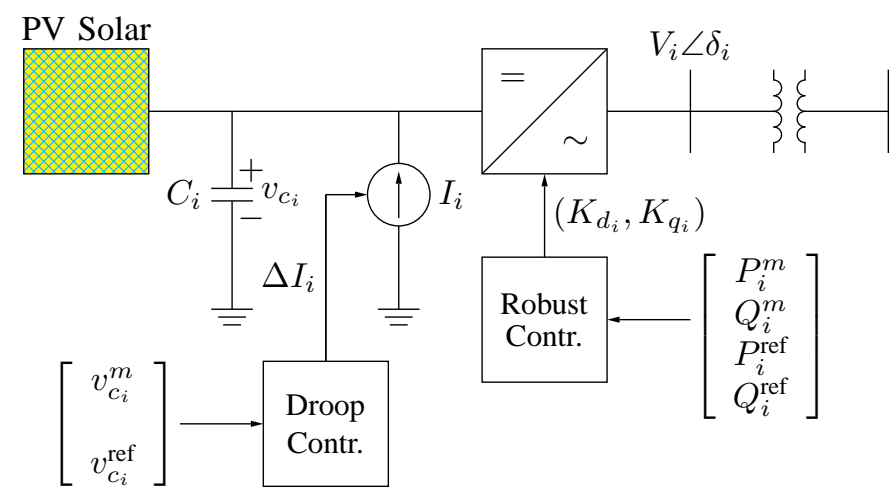

Fig. 4. PV \& Battery

\section{B. PV and BES Control}

A PV unit with a battery (represented as a current source) is shown in Fig. 4. The control laws for the PV unit are given as (superscript $m$ is used for measured values and $p$ for the PV unit):

$$
\begin{gathered}
\Delta I_{p}=-k_{I_{p}} \int\left(v_{c}^{\mathrm{ref}}-v_{c}^{\mathrm{m}}\right) d t \quad \text { (Battery Control) } \\
\Delta \delta_{p}=k_{\delta_{p}} \int\left(P_{p}^{\mathrm{ref}}-P_{p}^{\mathrm{m}}\right) d t
\end{gathered}
$$

$v_{c}^{\text {ref }}$ is obtained from the Maximum Power Point Tracking (MPPT) algorithm. In general $P_{p}^{\text {ref }}$ will be the predicted maximum power point based on solar radiance and $P_{p}^{\text {ref }}$ and $Q_{p}^{\text {ref }}$ are controlled using the robust controllers in [19]. In the voltage control mode of $\mathrm{PV}$ units, $P_{\mathrm{p}}$ and $Q_{\mathrm{p}}$ are controlled by the amplitude of the VSC terminal voltage. The error signals $P_{\text {ref }_{p}}-P_{\mathrm{p}}$ and $Q_{\text {ref }_{p}}-Q_{\mathrm{p}}$ are fed to the controller which produces $d$ - and $q$-axis components of the VSC current at their respective reference values which are again processed to get reference voltage components. The battery, shown as a current source in Fig. 4, charges or discharges to keep the capacitor voltage at $v_{d c}^{\text {ref }}$.

The values of the parameters mentioned above $k_{I_{w}}, k_{\delta_{w}}$, $k_{q_{w}}, k_{I_{p}}$ and $k_{\delta_{p}}$ are determined by choosing an integrating cost function that achieves near optimal time control performance. This is achieved by forming the product of the time and error in the cost function as shown below

$$
J=\left[\sum_{t=t_{0}}^{t_{f}}\left(t-t_{0}\right) \cdot W \cdot|E|\right]
$$

where $t_{0}$ and $t_{f}$ are the starting and ending times for calculating control performance, $W$ is a weighting matrix and $E=\left[\Delta v_{d c_{w}}, \Delta P_{w}, \Delta Q_{w}, \Delta v_{d c_{p}}, \Delta P_{p}\right]$ is the absolute error matrix; $\Delta P$ and $\Delta Q$ represent the error between the real and reactive power references and measurements and $\Delta v_{d c}$ is the voltage deviation from its nominal value.

The performance of the designed controller during an islanded mode is demonstrated in the following section.

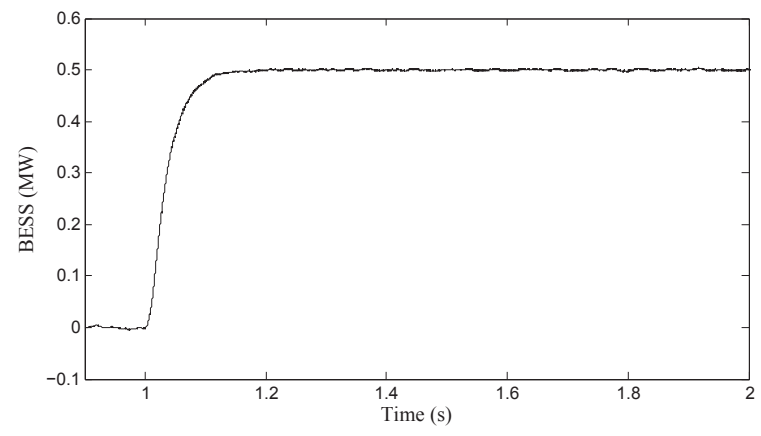

Fig. 5. Real power output of BESS unit during islanding.

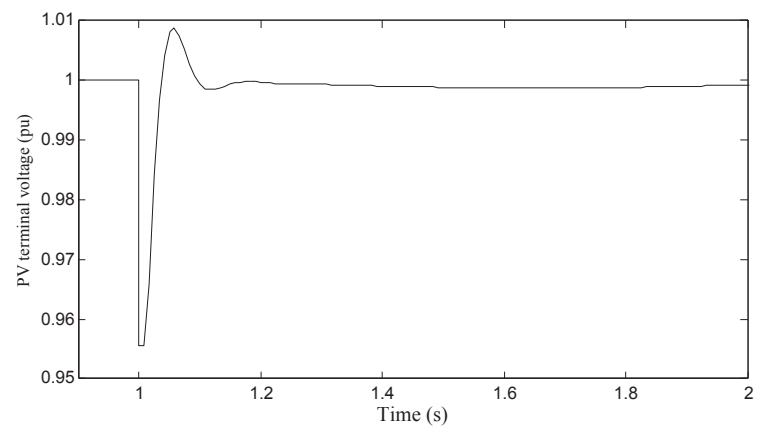

Fig. 6. Terminal voltage of PV unit during islanding.

\section{Controller Performance Evaluation}

The test microgrid in [22] is used for controller performance evaluation. The system consists of three $13.8 \mathrm{kV}$ feeders which are connected to the grid through a $69 \mathrm{kV}$ radial line. The total load of the system is 7.3 MW and 3.97 MVAr.

The PV unit is connected to the system via the VSC. The stator of the DFIG is connected directly to the grid and the rotor via a VSC. The rating of the PV unit is $3.0 \mathrm{MW}$ and the DFIG is $3.5 \mathrm{MW}$. Both the PV unit and the DFIG are connected to a battery energy storage system. The rating of each battery is $0.6 \mathrm{MWh}$. The DC-link voltage is $1200 \mathrm{~V}$ and capacitor is $10000 \mu \mathrm{F}$. Five-hundred and sixty-two $2.135 \mathrm{~V}$ lead-acid batteries are connected in series to get the desired voltage. Maximum charging or discharging current is $5 \mathrm{kA}$. Each distribution line is represented by lumped series RL branches. During the islanded mode the remaining balance of $1 \mathrm{MW}$ power is supplied by the BES.

The weighting matrix in (19) is chosen as $[0.5,1.0,1.0,0.5,1.0]$; the obtained control parameters for the wind turbines are $k_{I_{w}}=13.25, k_{\delta_{w}}=8, k_{v_{w}}=20$ and for the PV unit are $k_{I_{p}}=5, k_{\delta_{p}}=30$. Droop controllers are tuned first and then their dynamics are included in the robust controller design.

Initially the microgrid is working in a grid-connected mode, drawing $1 \mathrm{MW}$ of power from the grid, and the performance of the designed controller is investigated for a pre-planned islanding. At $1 \mathrm{~s}$, an intentional islanding command is applied to the $69 \mathrm{kV}$ line breakers and the batteries are switched on so that they share the remaining balance of $1 \mathrm{MW}$ of power. Figures 5 and 6 show the real power output of the battery 


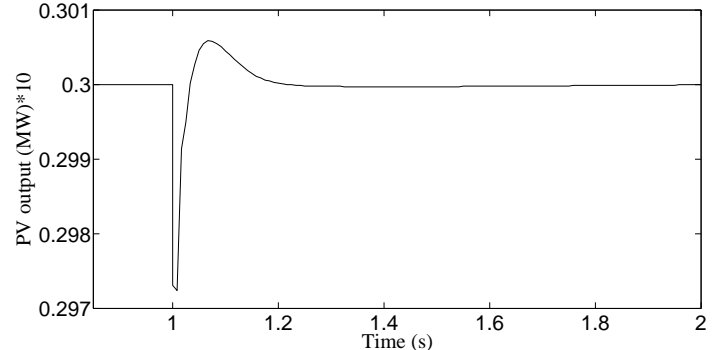

Fig. 7. Real power output of PV unit during islanding.

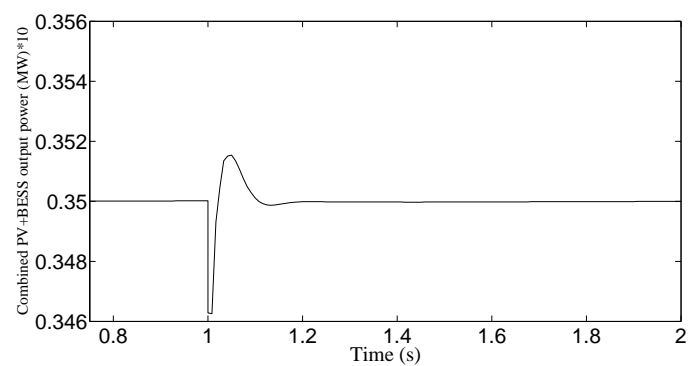

Fig. 8. Combined real power output of PV and BESS units during islanding.

energy storage system (BESS) and the terminal voltage of the PV unit, respectively. The wind generator and PV unit continue to operate at their optimum value. The real output power of the PV unit is shown in Fig. 7 the combined output power of the PV unit and BESS is shown in Fig. 8.

Both the DER units control their reactive power to control the terminal voltage. The voltage disturbance associated with mode transition is eliminated by the voltage controller. Both the phase angle and magnitude of the controlled voltage are quickly modulated by the proposed controller in order to reject the voltage and power angle disturbances. The damping provided by the robust power sharing controller yields a well damped power sharing performance. From this study it is clear that the proposed controller can ensure stability and damped transient performance during switching from grid-connected to islanded mode.

\section{Conclusion}

For ideal inverter connected voltage sources droop control can help in sharing real and reactive power. The control design is relatively simple owing to the restricted sensor and controller dynamic interaction. The challenge is to include generation and load dynamics, with their controls, and guarantee stability of islanded microgrids. Energy storage systems can be used to support DERs to achieve robust control to maintain stability for large disturbances and droop control for effective power sharing.

\section{REFERENCES}

[1] R.H. Lasseter. Microgrids. In Power Engineering Society Winter Meeting. IEEE, volume 1, pages 305-308, 2002.

[2] R.H. Lasseter. Smart distribution: Coupled microgrids. Proceedings of the IEEE, 99(6):1074-1082, June 2011.
[3] R.H. Lasseter, J.H. Eto, B. Schenkman, J. Stevens, H. Vollkommer, D. Klapp, E. Linton, H. Hurtado, and J. Roy. CERTS microgrid laboratory test bed. Power Delivery, IEEE Transactions on, 26(1):325332, Jan. 2011.

[4] K.. De Brabandere, B.. Bolsens, J.. Van den Keybus, A.. Woyte, J.. Driesen, and R.. Belmans. A voltage and frequency droop control method for parallel inverters. Power Electronics, IEEE Transactions on, 22(4):1107-1115, July 2007.

[5] J.M. Guerrero, M. Chandorkar, T. Lee, and P.C. Loh. Advanced control architectures for intelligent microgrids-Part I: Decentralized and hierarchical control. Industrial Electronics, IEEE Transactions on, 60(4):1254-1262, April 2013.

[6] J.M. Guerrero, Poh Chiang Loh, Tzung-Lin Lee, and M. Chandorkar. Advanced control architectures for intelligent microgrids-Part II: Power quality, energy storage, and ac/dc microgrids. Industrial Electronics, IEEE Transactions on, 60(4):1263-1270, April 2013.

[7] N.W.A. Lidula and A.D. Rajapakse. Microgrids research: A review of experimental microgrids and test systems. Renewable and Sustainable Energy Reviews, 15(1):186-202, 2011.

[8] Brian Johnson, Ali Davoudi, Patrick Chapman, and Peter Sauer. A unified dynamic characterization framework for microgrid systems Electric Power Components and Systems, 40(1):93-111, 2011.

[9] Jinwei He and Yun Wei Li. Analysis, design, and implementation of virtual impedance for power electronics interfaced distributed generation. Industry Applications, IEEE Transactions on, 47(6):2525-2538, Nov.-Dec. 2011.

[10] A.H. Etemadi, E.J. Davison, and R. Iravani. A decentralized robust control strategy for multi-DER microgrids-Part I: Fundamental concepts Power Delivery, IEEE Transactions on, 27(4):1843-1853, Oct. 2012.

[11] A.H. Etemadi, E.J. Davison, and R. Iravani. A decentralized robust control strategy for multi-DER microgrids-Part II: Performance evaluation. Power Delivery, IEEE Transactions on, 27(4):1854-1861, Oct. 2012.

[12] A. Tuladhar, Hua Jin, T. Unger, and K. Mauch. Control of parallel inverters in distributed AC power systems with consideration of line impedance effect. Industry Applications, IEEE Transactions on, 36(1):131-138, Jan/Feb 2000.

[13] Jinwei He and Yun Wei Li. An enhanced microgrid load demand sharing strategy. Power Electronics, IEEE Transactions on, 27(9):3984-3995, Sept. 2012.

[14] Qing-Chang Zhong. Robust droop controller for accurate proportional load sharing among inverters operated in parallel. Industrial Electronics, IEEE Transactions on, 60(4):1281-1290, April 2013.

[15] C.K. Sao and P.W. Lehn. Autonomous load sharing of voltage source converters. Power Delivery, IEEE Transactions on, 20(2):100 -1016, April 2005.

[16] M.J. Erickson, T.M. Jahns, and R.H. Lasseter. Comparison of PV inverter controller configurations for CERTS microgrid applications. In Energy Conversion Congress and Exposition (ECCE), 2011 IEEE, pages 659-666, Sept. 2011.

[17] S.J. Chiang and J.M. Chang. Parallel control of the UPS inverters with frequency-dependent droop scheme. In Power Electronics Specialists Conference, 2001. PESC. 2001 IEEE 32nd Annual, volume 2, pages 957-961, 2001

[18] R.M. Kamel, A. Chaouachi, and K. Nagasaka. Three control strategies to improve the microgrid transient dynamic response during isolated mode: A comparative study. Industrial Electronics, IEEE Transactions on, 60(4):1314-1322, April 2013.

[19] M. A. Mahmud, H. R. Pota, and M. J. Hossain. Dynamic stability of three-phase grid-connected photovoltaic system using zero dynamic design approach. IEEE Journal of Photovoltaics, 2(4):564-571, October 2012.

[20] M. J. Hossain, T. K. Saha, N. Mithulananthan, and H. R. Pota. Control strategies for augmenting LVRT capability of DFIGs in interconnected power systems. IEEE Transactions on Industrial Electronics, 60(6):2510 -2522, June 2013.

[21] N. K. Roy, H. R. Pota, and M. J. Hossain. Reactive power management of distribution networks with wind generation for improving voltage stability. Renewable Energy, 58:85-94, October 2013.

[22] F. Katiraei, M.R. Iravani, and P.W. Lehn. Micro-grid autonomous operation during and subsequent to islanding process. Power Delivery, IEEE Transactions on, 20(1):248-257, 2005. 